\title{
Effects of $\gamma$-Polyglutamic Acid on Blood Glucose and Caecal Short Chain Fatty Acids in Adult Male Mice
}

\author{
Motoi Tamura*, Sachiko Hori, Atsuko Inose, Masuko Kobori \\ Food Function Division, Food Research Institute of National Agriculture and Food Research Organization Tsukuba, \\ Ibaraki, Japan \\ Email: *motoita@affrc.go.jp
}

How to cite this paper: Tamura, M., Hori, S., Inose, A. and Kobori, M. (2020) Effects of $\gamma$-Polyglutamic Acid on Blood Glucose and Caecal Short Chain Fatty Acids in Adult Male Mice. Food and Nutrition Sciences, 11, 8-22.

https://doi.org/10.4236/fns.2020.111002

Received: August 27, 2019

Accepted: January 4, 2020

Published: January 7, 2020

Copyright () 2020 by author(s) and Scientific Research Publishing Inc. This work is licensed under the Creative Commons Attribution International License (CC BY 4.0).

http://creativecommons.org/licenses/by/4.0/

(c) (i) Open Access

\begin{abstract}
$\gamma$-Polyglutamic acid ( $\gamma$-PGA) is a major component of Natto. We hypothesized that $\gamma$-PGA could reduce postprandial glucose rise and plasma glucose levels. Mice were fed a $0.1 \% \gamma$-PGA-containing diet or control diet for 91 days. Maltose and starch tolerance tests were performed, and plasma lipids, glucose levels, and caecal short chain fatty acids (SCFAs) were measured. Mice were co-administered $\gamma$-PGA and starch to suppress the initial rise in blood glucose levels. Blood glucose levels at $15 \mathrm{~min}$ were significantly lower in the PGA group than in the Con group $(P<0.05)$. The plasma glucose level and NEFA level were also significantly lower in the PGA group $(P<0.05)$, and caecal acetic acid/total caecal SCFAs ratio was significantly increased in the PGA group $(P<0.05)$. Significant negative correlations existed between the caecal acetic acid/propionic acid ratio and the weight of visceral fat/BW $(\mathrm{r}=$ $-0.57, P=0.0318$ ). Our results suggest that $\gamma$-PGA may effectively prevent metabolic syndrome by lowering blood glucose levels.
\end{abstract}

\section{Keywords}

$\gamma$-Polyglutamic Acid, Mice, Blood Glucose, Short Chain Fatty Acids

\section{Introduction}

Natto is a traditional Japanese fermented soy food. $\gamma$-Polyglutamic acid ( $\gamma$-PGA) is one of the main components of Natto. $\gamma$-PGA is an anionic polyamide and glutamic acid is polymerized via $\gamma$-amide linkages. Figure 1 shows the chemical structure of $\gamma$-polyglutamic acid. $\gamma$-PGA is produced by some strains of Bacillus [1]. Natto contains $\gamma$-PGA produced by Bacillus subtilis (natto) during fermentation 


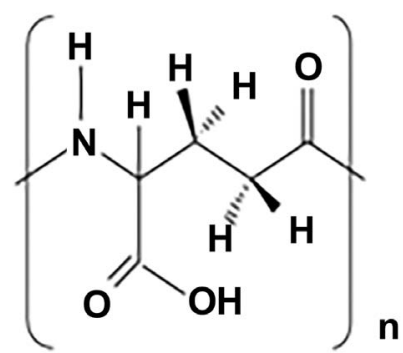

Figure 1. Chemical structure of $\gamma$-polyglutamic acid.

process [2]. High-performance liquid chromatography (HPLC), sodium dodecyl sulfate-polyacrylamide gel electrophoresis, and the cetyltrimethylammonium bromide (CTAB)-dependent spectrophotometric assay are used for quantification of $\gamma$-PGA [3].

A number of beneficial effects of $\gamma$-PGA have been previously reported. In mice, high-fat diets containing 3\% $\gamma$-PGA significantly increased the serum HDL-cholesterol and significantly decreased the serum TG as compared to levels observed in the mice fed a high-fat control diet [4]. Inhibition of intestinal high-fat absorption is one of the mechanisms of improved lipid metabolism of the mice fed the high-fat diet [4]. Diets containing high molecular weight $\gamma$-PGA were fed to rats, and their estimated adiposity and lipid metabolism were then measured. Findings from this research suggest that, in rats, dietary supplementation with high molecular weight $\gamma$-PGA may act as a hypocholesterolemic agent [5]. Experiments performed in vitro suggest that $\gamma$-PGA induces apoptosis in TPA-induced HT-29 human colorectal cancer cells and enhances apoptosis in colorectal cancer cells [6].

Cheonggukjang is a Korean food made from soy beans fermented by Bacillus, and it is known to contain $\gamma$-PGA. Frequent consumption of Cheonggukjang has been suggested to prevent several obesity-related factors [7]. It has been reported that soybeans fermented by Bacillus spp. possess high $\gamma$-PGA content that can improve insulin-sensitizing activity in adipocytes and neuronal cells [8]. From available reports, $\gamma$-PGA appears to be effective for improving lipid metabolism and glucose metabolism. In research using healthy male volunteers, Natto administration significantly lowered blood glucose levels as compared to levels observed after control meals [9]. Natto appears to reduce postprandial glucose rise, however, Natto contains a number of food components such as dietary fiber, soy isoflavones, and soy protein. Given these additional components, the main factor responsible for the blood glucose lowering effect observed after Natto intake remains unknown.

We hypothesized that $\gamma$-PGA, one of the main components of Natto, is responsible for the reduction in postprandial glucose rise and that dietary $\gamma$-PGA is responsible for the plasma glucose-lowering effects. In this study, we tested these effects in vivo using adult male mice. 


\section{Materials and Methods}

\subsection{Materials}

$\gamma$-Polyglutamic acid ( $\gamma$-PGA) was purchased from Meiji Food Materia Co. Ltd. (Tokyo, Japan). The molecular weight of $\gamma$-PGA ranged from $600 \mathrm{kDa}$ to 1500 $\mathrm{kDa}$. The purity of $\gamma$-PGA was greater than $70 \%$.

\subsection{Treatments of Animals}

Male Jcl:ICR mice (6 weeks old) were purchased from CLEA Japan, Inc. (Tokyo, Japan). All mice were specific pathogen-free (SPF) and were randomly subdivided into two groups of seven animals each. The mice were housed individually in suspended stainless-steel cages with wire mesh bottoms in a room maintained at $24^{\circ} \mathrm{C} \pm 0.5^{\circ} \mathrm{C}$ under a relative humidity of $65 \%$ with 12 -h periods of light and darkness. The mice were fed the AIN-93G diet for 14 days. After 14 days, the diet was replaced with a $0.1 \% \gamma$-PGA - containing diet (PGA group) or a control diet (Con group) for 91 days. Table 1 presents the composition of each diet. The concentration $(0.1 \%)$ of $\gamma$-PGA offered to the mice is compatible with human consumption. After 28 days from the start of the experimental diet, the maltose tolerance test was performed. After 56 days from start of feeding of the experiment diet, the starch tolerance test was performed. After the experimental diet period, the mice were euthanized by collecting blood from the heart during isoflurane anesthesia, and blood samples were taken from the heart and placed in heparinized tubes. The plasma was separated from whole blood by centrifugation and stored at $-80^{\circ} \mathrm{C}$ until analysis of plasma lipids. The liver, visceral fat, and caecal contents were collected. Caecal contents were stored at $-80^{\circ} \mathrm{C}$ until use in caecal short chain fatty acids analysis. The liver samples and visceral fat were weighed. All procedures involving mice in this study were approved by the

Table 1. Composition of the experimental diet.

\begin{tabular}{ccc}
\hline Ingredient $(\mathrm{g} / \mathrm{kg}$ diet$)$ & Con diet & PGA diet \\
\hline Com starch & 297.486 & 297.486 \\
Casein & 200 & 199 \\
$\alpha$-Corn starch & 132 & 132 \\
Sucrose & 200 & 200 \\
Soy bean oil & 70 & 70 \\
Cellulose & 50 & 50 \\
Mineral mix (AN-93G-Mix) & 35 & 35 \\
Vitamin mix (AIN-93-Mix) & 10 & 10 \\
L-Cystine & 3 & 3 \\
Choline bitartrate & 2.5 & 2.5 \\
Tert-butylhydroquinone & 0.014 & 0.014 \\
$\gamma$-Polyglutamic acid & & 1 \\
\hline
\end{tabular}


Animal Care Committee of Food Research Institute (Tsukuba, Japan) in accordance with the "Guidelines for Animal Care and Experimentation" of Food Research Institute, National Agriculture and Food Research Organization (Tsukuba, Japan).

\subsection{Maltose and Starch Tolerance Test}

After 28 days from the start of the experiment diet, the maltose tolerance test was performed. As the relatively high concentration of $\gamma$-PGA was expected to suppress the increase in blood glucose level, we used $8 \% \gamma$-PGA in this experiment. All mice have fasted for $12 \mathrm{hrs}$. Immediately after measurement of initial blood glucose levels, maltose solution ( $1 \mathrm{~g} / \mathrm{kg}$ body weight) containing $8 \% \gamma$-PGA was orally administered to the PGA group using a feeding needle. Maltose solution ( $1 \mathrm{~g} / \mathrm{kg}$ body weight) was orally administered to the Con group using a feeding needle. The blood glucose levels were measured at $15 \mathrm{~min}, 30 \mathrm{~min}, 60 \mathrm{~min}$, and 120 min after maltose administration. The blood glucose levels were measured in blood collected from the tail vein using the One Touch Ultra View ${ }^{\otimes}$ blood glucose monitoring system (Johnson \& Johnson Lifescan, Inc., Milpitas, CA, USA). For measurements of blood glucose, the value obtained from the maltose tolerance test was subtracted from the initial blood glucose levels. After 56 days from the start of the experiment diet, the starch tolerance test was performed. All mice have fasted for $12 \mathrm{hrs}$. Immediately after measurement of initial blood glucose levels, starch solution ( $1 \mathrm{~g} / \mathrm{kg}$ body weight) containing $8 \% \gamma$-PGA was orally administered to the PGA group using a feeding needle. Starch solution $(1 \mathrm{~g} / \mathrm{kg}$ body weight) was orally administered to the Con group using a feeding needle. The blood glucose levels were measured using blood collected from the tail vein, by the One Touch Ultra View ${ }^{\oplus}$ blood glucose monitoring system (Johnson \& Johnson Lifescan, Inc., Milpitas, CA, USA) at $15 \mathrm{~min}, 30 \mathrm{~min}, 60 \mathrm{~min}$, and 120 min after starch administration. For measurements of blood glucose, the value obtained from the starch tolerance test was subtracted from the initial blood glucose levels.

\subsection{Measurement of Plasma Cholesterol, Triglyceride, Nonesterified Fatty Acid, HDL-Cholesterol, and Glucose Levels}

The following tests were performed using kits obtained from FUJIFILM Wako Pure Chemical Corporation (Osaka, Japan). Total plasma cholesterol concentrations were measured using a cholesterol E-test kit based on cholesterol oxidase. Plasma triglyceride concentrations were determined using a triglyceride E-test kit based on the glycerol-3-phosphate oxidase method. Plasma nonesterified fatty acid (NEFA) concentrations were measured using a NEFA C-test kit utilizing acyl-coenzyme A (CoA) synthase, acyl-CoA oxidase, peroxidase. Plasma HDLcholesterol concentrations were measured using a HDL-cholesterol E-test kit. Plasma glucose concentrations were quantified using a glucose CII-test kit involving glucose oxidase and peroxidase. 


\subsection{Measurement of Caecal Short Chain Fatty Acids}

Caecal contents were diluted in distilled water, vortexed for $30 \mathrm{~s}$, and then centrifuged. Supernatants were analyzed using the short chain fatty acid analysis kit (YMC Co. Ltd., Kyoto, Japan). This kit is based on the method of Miwa et al. [10] [11]. In this kit, by converting the carboxyl group of the short chain fatty acid to 2-nitrophenylhydrazine (2-NPH), high-sensitivity detection in the ultraviolet and visible regions is possible.

Crotonic acid was used as the internal standard. Crotonic acid was added diluted caecal contents. A total of $150 \mu \mathrm{L}$ of diluted caecal contents was added to $100 \mu \mathrm{L}$ of 2-nitrophenylhydrazine hydrochloride $(2-\mathrm{NPH} \cdot \mathrm{HCl})$ solution and 100 $\mu \mathrm{L}$ of working 1-ethyl-3-(3-dimethylaminopropyl)-carbodiimide hydrochloride $(1-\mathrm{EDC} \cdot \mathrm{HCl})$ solution. The mixture was incubated at $60^{\circ} \mathrm{C}$ for $20 \mathrm{~min}$. After addition of $100 \mu \mathrm{L}$ of potassium hydroxide solution, the mixture further incubated for $15 \mathrm{~min}$. After cooling in room temperature, $2 \mathrm{~mL}$ of phosphate buffer- $0.5 \mathrm{M}$ hydrochloric acid was added to neutralize the solution. The mixture was washed with $2.5 \mathrm{~mL}$ of $\mathrm{n}$-hexane to remove the long-chain fatty acid hydrazides and interfering substances. About $1.5 \mathrm{~mL}$ of aliquot of the aqueous layer was taken. The short chain fatty acid hydrazides in aliquot of the aqueous layer were extracted with $2.5 \mathrm{~mL}$ of diethyl ether. The ether layer was taken. The ether layer was evaporated with a stream of nitrogen gas. The residue was dissolved in 200 $\mu \mathrm{L}$ of methanol. For HPLC analysis, $20 \mu \mathrm{L}$ of each preparation was injected into a $250 \times 6.0 \mathrm{~mm}$ YMC-Pack FA (YMC, Kyoto, Japan). To detect the hydrazides of short chain fatty acids, a photodiode array detector (MD-1515; JASCO, Co., Ltd., Tokyo, Japan) was used to monitor the spectral data from 200 to $448 \mathrm{~nm}$ for each peak. Acetic acid, propionic acid, butyric acid, isovaleric acid, and valeric acid were used as standard samples. Crotonic acid was used as the internal standard. The spectral data at $400 \mathrm{~nm}$ was used to quantify the hydrazides of short chain fatty acid. The mobile phase consisted of acetonitrile/methanol/water (30:16:54, $\mathrm{v} / \mathrm{v} / \mathrm{v}, \mathrm{pH}$ 4.5). The running conditions of HPLC were a column temperature of $50^{\circ} \mathrm{C}$ and a flow rate of $0.9 \mathrm{~mL} / \mathrm{min}$. HPLC chromatogram of acetic acid hydrazide, propionic acid hydrazide, butyric acid hydrazide, crotonic acid hydrazide, isovaleric acid hydrazide, and valeric acid hydrazide in standard sample obtained from at $400 \mathrm{~nm}$ is shown in Figure 2.

\subsection{Statistics}

Data are expressed as the mean \pm standard error (SE). All data were analyzed using Sigma Plot 11 (Systat Software, Inc., San Jose, CA, USA). The data were analyzed by the Student's t-test, Mann-Whitney rank sum test, and/or Pearson Product-Moment Correlation. Statistical significance was confirmed at p-value < 0.05 .

\section{Results}

\subsection{General Observations}

No significant differences were observed between the PGA and Con groups in 


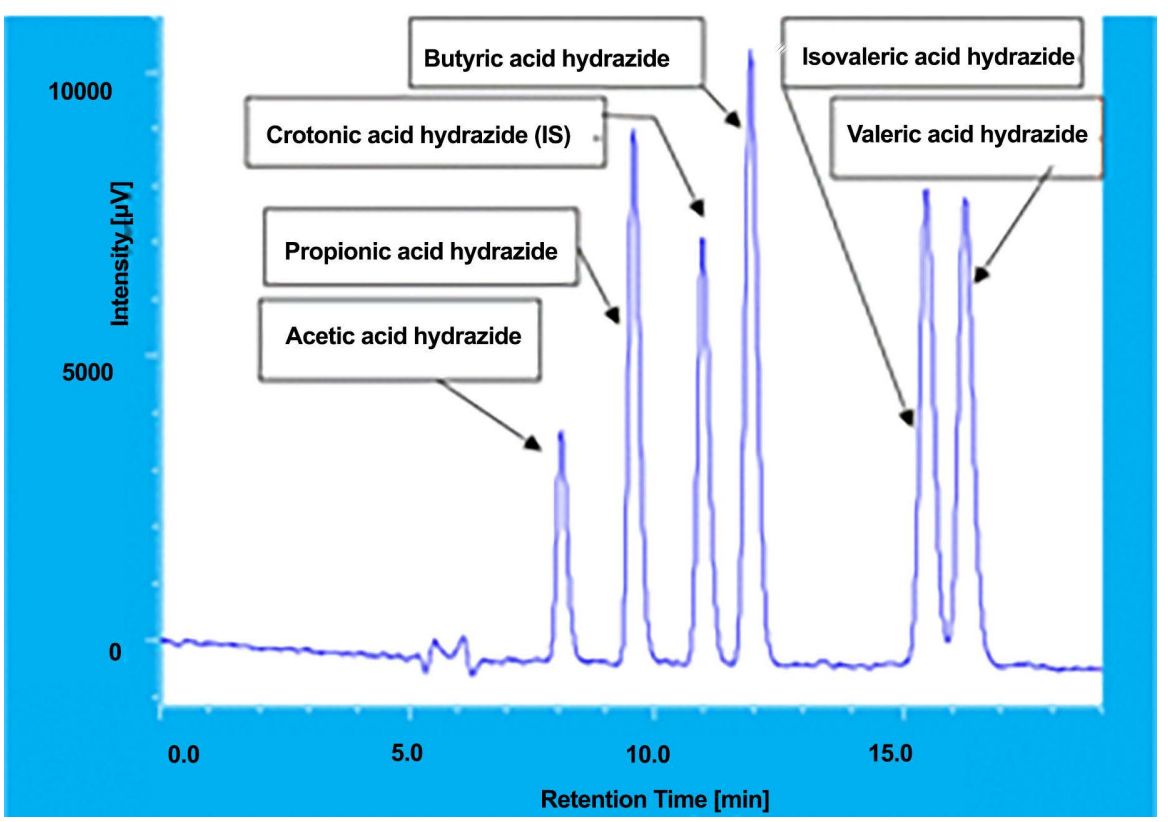

Figure 2. HPLC chromatogram of acetic acid hydrazide, propionic acid hydrazide, butyric acid hydrazide, crotonic acid hydrazide, isovaleric acid hydrazide, and valeric acid hydrazide in standard sample obtained from at $400 \mathrm{~nm}$.

regard to final body weight (PGA [48.7 $\mathrm{g} \pm 1.4$ ], Con [50.3 $\mathrm{g} \pm 2.0$ ], liver weight (PGA [1.99 g \pm 0.11$]$, Con [2.25 g \pm 0.13 ], and caecal contents (PGA [0.13 g \pm $0.02]$, Con $[0.15 \mathrm{~g} \pm 0.02]$. The average visceral fat per final body weight in the PGA group $(0.066 \pm 0.004)$ was lesser than that in the Con group $(0.081 \pm$ $0.010)$. The perigenital visceral fat per final body weight in the PGA group $(0.034$ $\pm 0.002)$ also tended to be lesser than that in the Con group $(0.046 \pm 0.006)(P=$ 0.097). There were no significant differences in food consumption (g/day) between the PGA group $(4.66 \pm 0.06)$ and the Con group $(4.67 \pm 0.07)$.

\subsection{Measurement of Plasma Cholesterol, Triglyceride, NEFA, HDL-Cholesterol, and Glucose Levels}

The plasma concentrations of triglyceride, total cholesterol, and HDL-cholesterol were investigated, and are presented in Table 2 . No significant differences were observed in the plasma concentrations of triglyceride, total cholesterol, and HDL-cholesterol between two groups. The plasma glucose levels (Figure 3) and the plasma nonesterified fatty acids (NEFA) levels (Figure 4), however, were significantly lower in the PGA group compared to those in the Con group $(P<$ $0.05)$.

\subsection{Maltose and Starch Tolerance Test}

The results of the maltose tolerance test are presented in Figure 5. All data are representative of values deducted from the blood glucose levels immediately prior to administration of maltose. In the PGA group, the average blood glucose levels measured at $15 \mathrm{~min}, 30 \mathrm{~min}, 60 \mathrm{~min}$, and $120 \mathrm{~min}$ after maltose loading 
Table 2. The concentrations of triglyceride (TG) (mg/dL), total cholesterol (Chol) $(\mathrm{mg} / \mathrm{dL})$ and HDL-cholesterol (HDL-Chol) $(\mathrm{mg} / \mathrm{dL})$ in plasma.

\begin{tabular}{cccc}
\hline & Chol $(\mathrm{mg} / \mathrm{dL})$ & TG $(\mathrm{mg} / \mathrm{dL})$ & HDL-Chol $(\mathrm{mg} / \mathrm{dL})$ \\
\hline Con group & $133.7 \pm 15.3$ & $94.9 \pm 13.2$ & $97.7 \pm 7.2$ \\
PGA group & $137.7 \pm 17.1$ & $82.3 \pm 15.2$ & $105.6 \pm 7.7$ \\
\hline
\end{tabular}

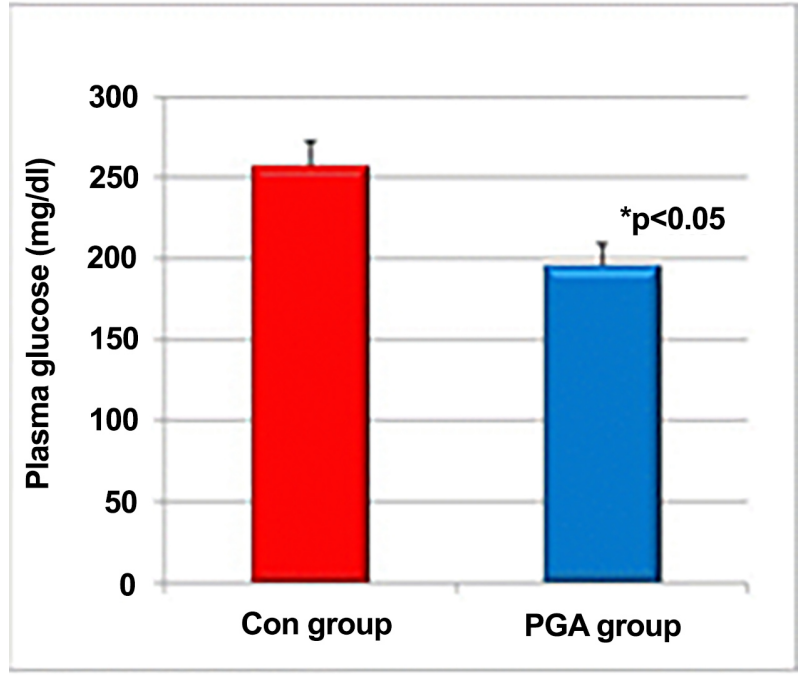

Figure 3. Plasma glucose concentrations in the PGA and the Con groups. ${ }^{\star} p<0.05$. Values are means $\pm \mathrm{SE}(\mathrm{n}=7)$.

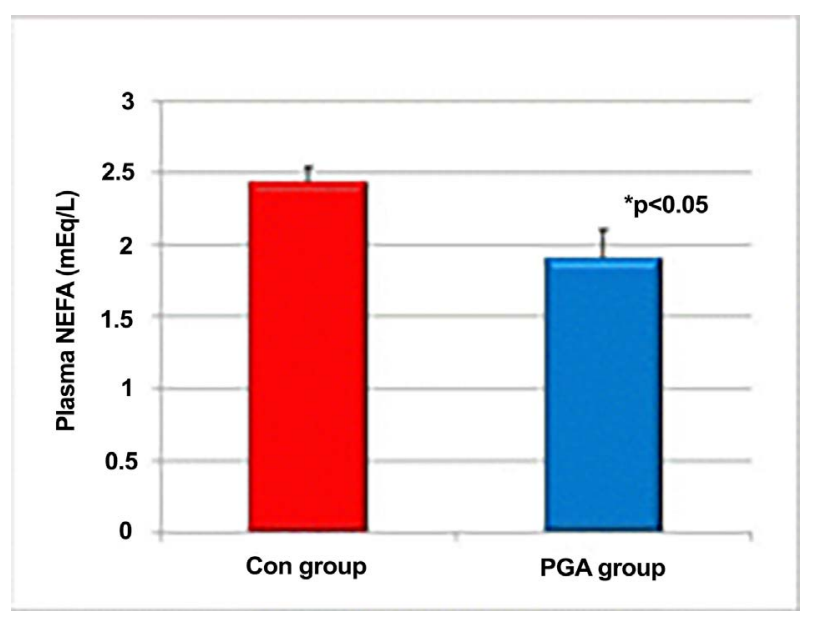

Figure 4. Plasma nonesterified fatty acids (NEFA) concentrations in the PGA and the Con groups. ${ }^{\star} p<0.05$. Values are means \pm SE $(n=7)$.

were lower than those in the Con group. Incremental AUC determined from 0 min to 120 min was lower in the PGA group than in the Con group $(P=0.086)$. The results of starch tolerance test are provided in Figure 6. All data are representative of values deducted from the blood glucose level immediately prior to administration of starch. Blood glucose levels at $15 \mathrm{~min}$ were significantly lower in the PGA group than in the Con group $(P<0.05)$. Co-administration of 


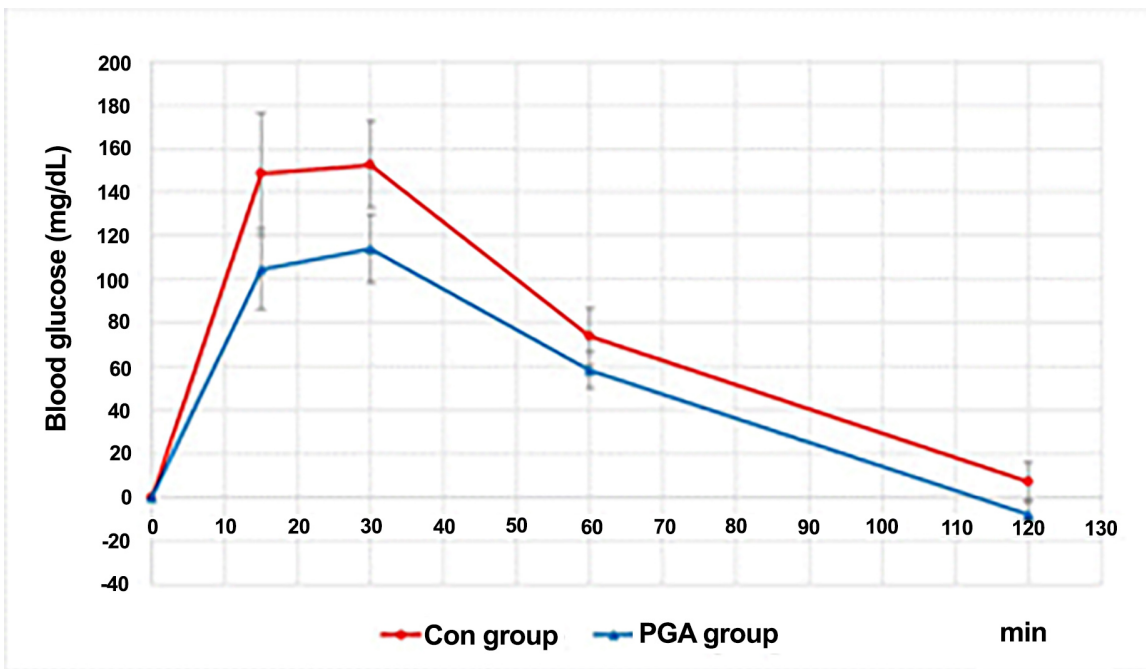

Figure 5. Blood glucose levels at $15 \mathrm{~min}, 30 \mathrm{~min}, 60 \mathrm{~min}$ and $120 \mathrm{~min}$ after maltose loading in the PGA group (blue line) and the Con group (red line). Values are means \pm SE (n $=7)$.

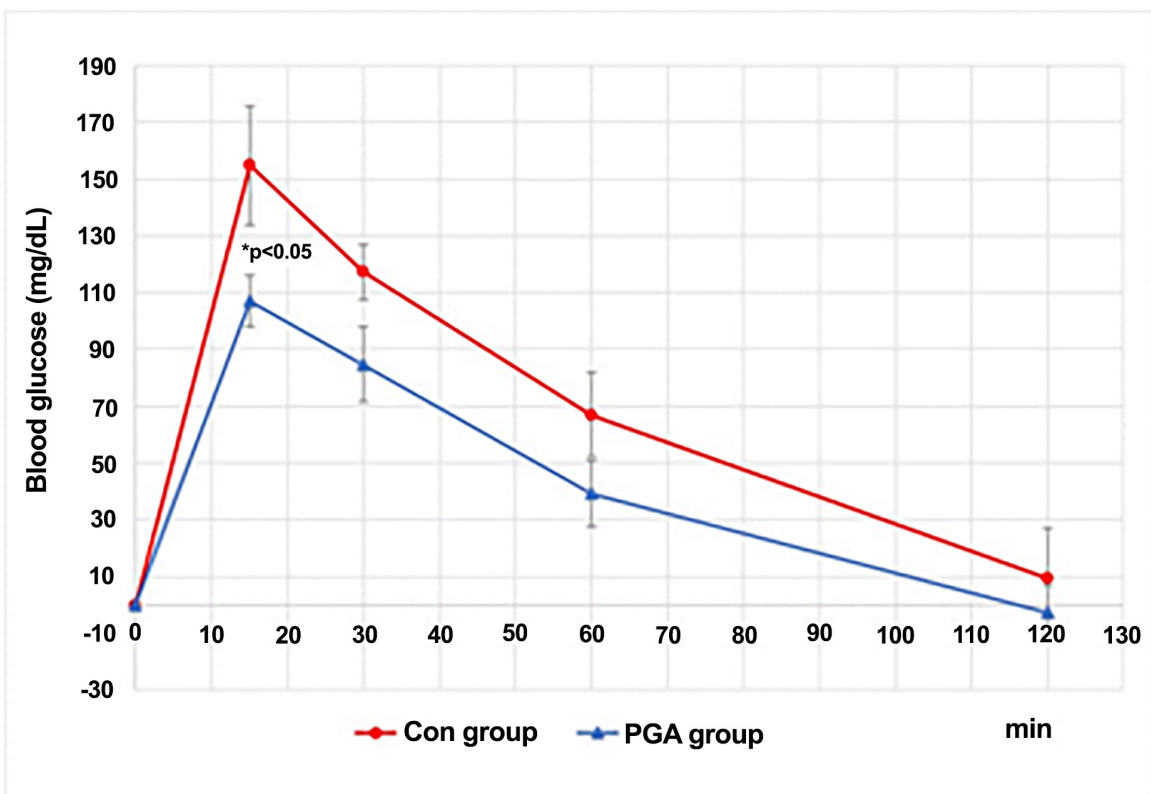

Figure 6. Blood glucose levels at $15 \mathrm{~min}, 30 \mathrm{~min}, 60 \mathrm{~min}$ and $120 \mathrm{~min}$ after starch loading in the PGA group (blue line) and the Con group (red line). Blood glucose levels at $15 \mathrm{~min}$ were significantly lower in the PGA group $\left({ }^{\star} p<0.05\right)$. Values are means $\pm \mathrm{SE}(\mathrm{n}=7)$. Blood glucose levels tended to be low in the PGA group.

$\gamma$-PGA and starch to mice is believed to suppress the initial rise in blood glucose levels. In the PGA group, the average blood glucose levels measured at $30 \mathrm{~min}$, $60 \mathrm{~min}$, and $120 \mathrm{~min}$ after starch loading were lower than those observed in the Con group. Incremental AUC determined from $0 \mathrm{~min}$ to $15 \mathrm{~min}, 0 \mathrm{~min}$ to 30 min, and 0 min to 60 min was significantly lower in the PGA group than in the Con group $(P<0.05)$. These results suggest that PGA decreases postprandial glucose rise in adult male mice. 


\subsection{Caecal Short Chain Fatty Acid Concentration}

The caecal short chain fatty acid concentrations observed in the two dietary groups are shown in Figure 7. The average caecal acetic acid concentration was higher in the PGA group than in the Con group. The average total caecal short chain fatty acid concentration (acetic acid+ butyric acid + propionic acid + valeric acid + isovaleric acid) was higher in the PGA group than in the Con group. The ratio of caecal acetic acid/total caecal short chain fatty acids was significantly greater in the PGA group $(P<0.05)$ than in the Con group (Figure 8). In contrast, the ratio of caecal butyric acid/total caecal short chain fatty acids was significantly lower in the PGA group $(P<0.05)$ (Figure 8$)$. These results suggest that the production rates of each of the short chain fatty acids differ between the two groups. The ratio of caecal acetic acid/propionic acid was related to the weight of visceral fat/BW. From the Pearson Product-Moment Correlation analysis, significant negative correlations were observed between the ratio of caecal acetic acid/propionic acid and the weight of visceral fat/BW $(r=-0.57)$ (Figure 9).

\section{Discussion}

Starch administration in combination with $\gamma$-PGA significantly decreased blood glucose levels $15 \mathrm{~min}$ at post-administration when compared to the control group. Co-administration of $\gamma$-PGA and starch to mice is thought to suppress the initial rise in blood glucose levels. Blood glucose levels tended to be low in the PGA groups after co-administration of $\gamma$-PGA and maltose to mice, however, no significant difference was observed in the blood glucose levels at $15 \mathrm{~min}$ post-administration when compared to levels observed in the control group $(P=0.216)$. It has been reported that in vitro, 3-hydroxy-3-methylglutaryl

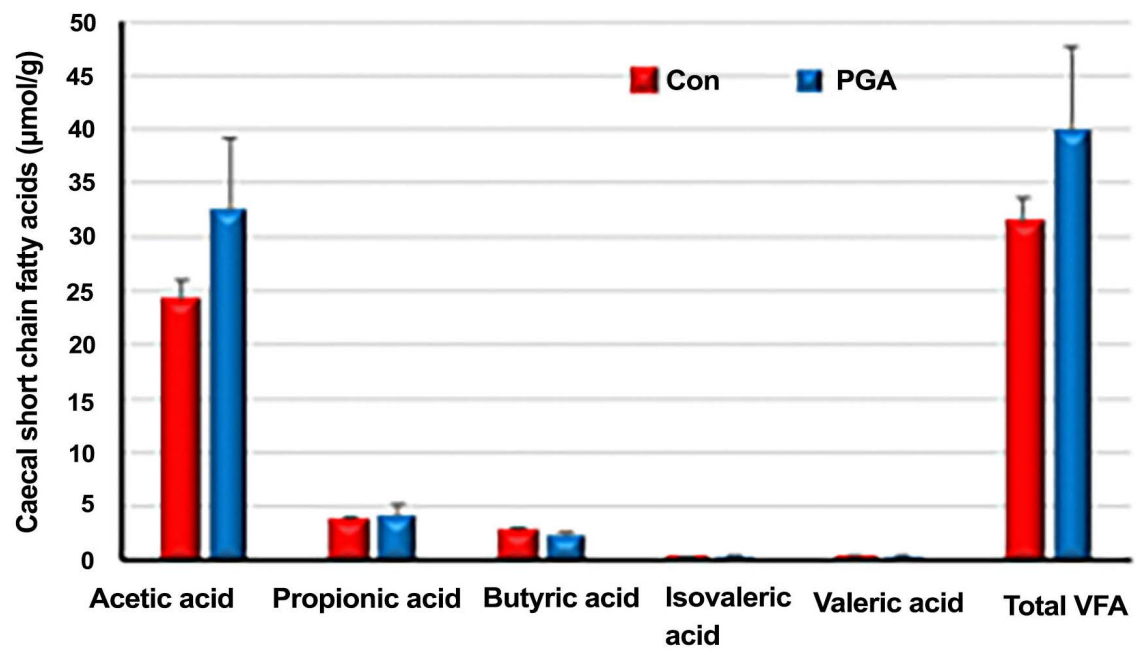

Figure 7. Caecal short chain fatty acids (SCFAs) concentration in the PGA and Con groups. Average total caecal short chain fatty acids concentration (acetic acid + butyric acid + propionic acid + valeric acid + isovaleric acid) was higher in the PGA group than in the Con group. VFA means short chain fatty acids. 


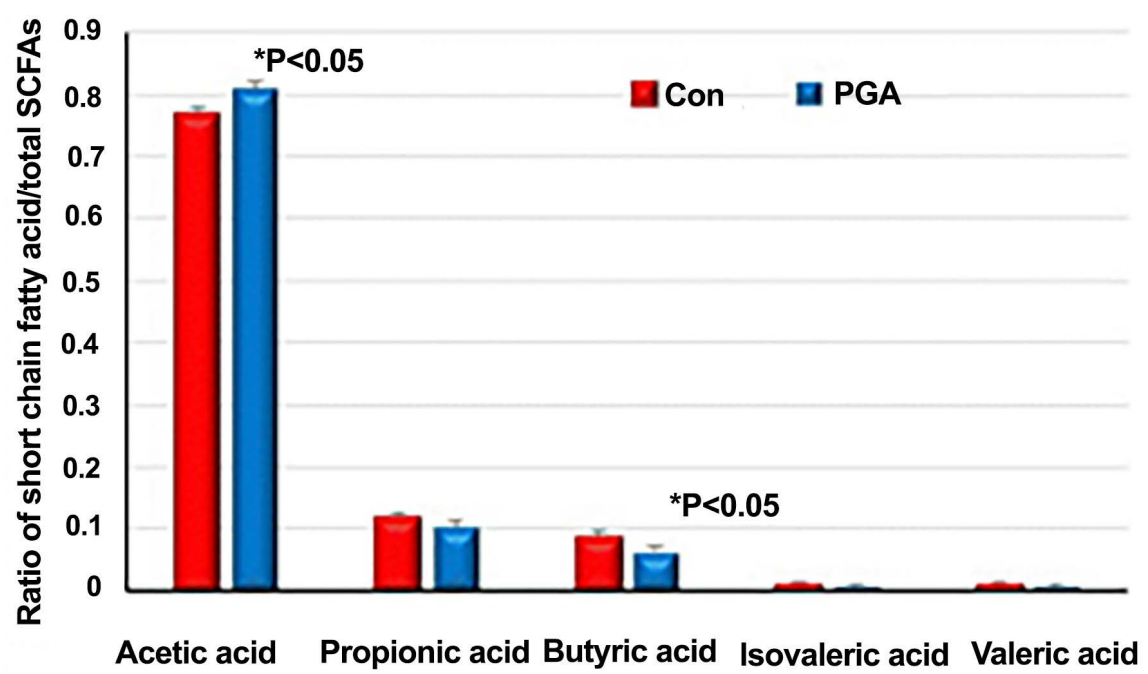

Figure 8. The ratio of caecal acetic acid/total SCFAs, caecal butyric acid/total SCFAs, caecal propionic acid/total SCFAs, caecal valeric acid/total SCFAs, caecal isovaleric ac$\mathrm{id} /$ total SCFAs in the PGA and Con groups. The ratio of caecal acetic acid/total caecal SCFAs is significantly greater in the PGA group $\left({ }^{\star} P<0.05\right)$. The ratio of caecal butyric acid/total caecal SCFAs is significantly lower in the PGA group $\left({ }^{\star} P<0.05\right)$. SCFAs means short chain fatty acids.

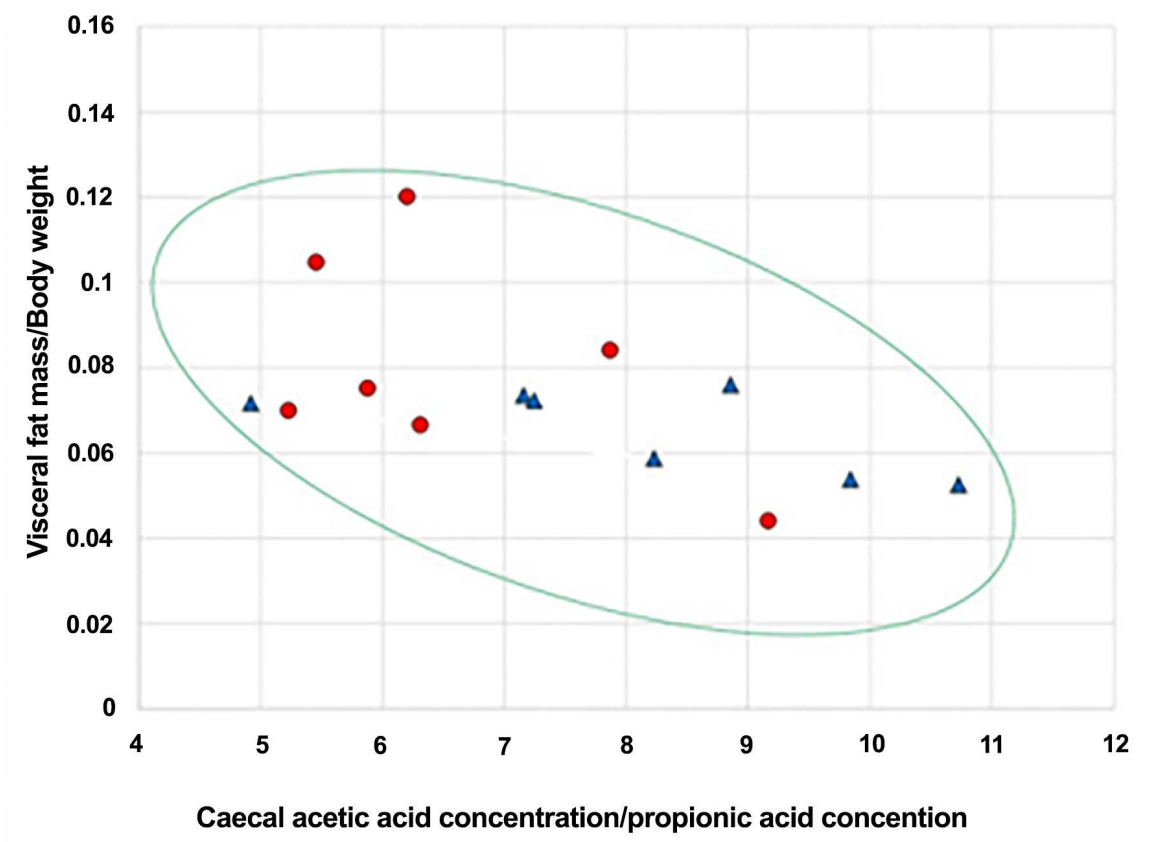

Figure 9. Correlation between the ratio of caecal acetic acid/propionic acid and the weight of visceral fat/BW. Pearson product-moment correlation coefficient between the ratio of caecal acetic acid/propionic acid and the weight of visceral fat/BW were analyzed. Significant negative correlations were observed between the ratio of caecal acetic ac$\mathrm{id} /$ propionic acid and the weight of visceral fat/BW $(\mathrm{r}=-0.57)(P=0.0318)$. Closed circle is the Con group. Closed triangle is the PGA group.

coenzyme-A (HMG-CoA) reductase activity can be suppressed by the addition of high molecular weight $\gamma$-PGA [5]. $\gamma$-PGA appears to exert inhibitory effects 
on enzyme activity. Inhibitory effects of $\gamma$-PGA targeting digestive enzymes may occur and partially contribute to the weak initial rise in blood glucose levels observed in the PGA group.

The viscosity of dietary fiber has been suggested to be important for improving glucose homeostasis [12]. It has been reported that intake of viscous $\mathcal{B}$-glucan can delay gastro emptying, and attenuation of the postprandial glucose response after $\mathcal{B}$-glucan intake has been reported in healthy volunteers [13]. Additionally, administration of viscous sodium-alginate with meals can reduce postprandial glucose and insulin concentrations in patients with Type 2 diabetes mellitus. Gastric emptying rates were lower in patients administered viscous sodium-alginate with meals than in patients provided with fiber-free meals, and glucose levels correlated with lower gastric emptying rates [14]. It has been reported that a high gastric emptying rate is related to higher blood glucose after meal intake [15]. Lower rates of gastric emptying correlated with lower postprandial blood glucose levels. In our results, plasma glucose levels were significantly lower in the PGA groups than in the Con groups as assessed by oral starch tolerance tests. Similar to dietary fiber, $\gamma$-PGA is also a viscous polymer. Given this, gastric emptying rates in the PGA group may be lower than in the Con group, and these slower gastric emptying rates may result in the reduced glucose levels detected by the oral starch administration test. Postprandial glucose levels are known to be related to chronic metabolic diseases such as obesity and type 2 diabetes mellitus [16]. Proper control of gastric emptying rates may allow for control of postprandial glycemia, and in turn, this may help to prevent obesity and the development of Type 2 diabetes mellitus. $\gamma$-PGA is a candidate food component that may aid in regulating postprandial glycemia. $\gamma$-PGA is a major component of Natto, and administration of this food significantly lowered blood glucose levels compared to control meals in the healthy male volunteers [9]. Our results suggest that the glucose-lowering effects of Natto can mainly be attributed to the $\gamma$-PGA present in Natto.

Plasma NEFA concentrations were significantly lower in the PGA group than in the Con group. Obesity is a risk factor for the development of Type 2 diabetes, and circulating non-esterified fatty acids (NEFA) chronically increases this risk [17]. In adipose tissue, NEFA are produced by the hydrolysis of triglycerides derived from the adipose tissue [18]. It has been suggested that plasma FFA levels are elevated in most obese subjects [19]. A positive association between NEFA and type 2 diabetes in adults has been reported [20] [21]. Lower plasma NEFA concentrations in the PGA group may reflect the lower visceral fat found in this group. It has been reported that the development of diabetes is increased with absolute and/or relative increases in visceral fat [22]. Type 2 diabetes and visceral fat are directly related to the presence of metabolic syndrome [23]. To reduce the risk of developing metabolic syndrome, it is important to reduce visceral fat and, in turn, reduce the incidence of Type 2 diabetes. Blood glucose levels are one of the diagnostic criteria for metabolic syndrome [24] and Type 2 diabetes. According to our results, plasma glucose levels were significantly lower in the PGA 
groups than in the Con group. Our results suggest that $\gamma$-PGA may be an important food component that can help prevent the incidence of the metabolic syndrome.

Short chain fatty acids are major end products of intestinal bacterial fermentation. Our results suggest that the production rates of each of the short chain fatty acids differ between the two groups. $0.1 \% \gamma$-PGA might affect the caecal short chain fatty acids production in mice. The cell membrane receptors GPR41 and GPR 43 are short chain fatty acid receptors. GPR41 is primarily activated by propionic acid and butyric acid, and GPR43 is typically activated by acetic acid and propionic acid [25]. It has been reported that GPR 43 recognizes excess energy by directly detecting short chain fatty acids, and this receptor also functions to suppress excess energy accumulation in adipose tissue, induce energy consumption in the direction of energy consumption, and maintain energy integrity within the body [26]. Our results indicate significant negative correlations between the ratio of caecal acetic acid/propionic acid and weight of visceral fat/BW ( $\mathrm{r}=-0.57, P=0.0318$ ). Negative correlations were also observed between the ratio of caecal acetic acid/total SCFAs and the weight of visceral fat/BW ( $\mathrm{r}=-0.44, P=0.110)$ and the weight of genital visceral fat $/ \mathrm{BW}(\mathrm{r}=$ $-0.49, P=0.075)$. Our results suggest that the production ratio of individual caecal SCFAs is related to visceral fat accumulation in mice. The visceral fat masses and perigenital visceral fat masses were lower in the PGA group compared to those of the Con group, and the ratio of caecal acetic acid/total SCFAs was significantly higher in the PGA group. These results suggest that the visceral fat-lowering effects of dietary PGA may partially occur through the modification of caecal short chain fatty acids.

\section{Conclusion}

Dietary $\gamma$-PGA reduces plasma glucose and NEFA levels. Based on the starch tolerance test, co-administration of $\gamma$-PGA and starch to mice suppressed the initial rise in blood glucose levels. Blood glucose levels at 15 min were significantly lower in the PGA group compared to those in the Con group $(P<0.05)$. The amount of perigenital fat per body weight tended to be lower in the PGA group than in the Con group. The production rates of each short chain fatty acid differ between the two groups, and significant negative correlations were observed between the ratio of caecal acetic acid/propionic acid and weight of visceral fat $/ \mathrm{BW}(\mathrm{r}=-0.57, \mathrm{P}=0.0318)$. Our results suggest that $\gamma$-PGA demonstrates the ability to prevent metabolic syndrome.

\section{Acknowledgements}

This work was supported by a grant from a commissioned project study titled "Research Development for Discovering of regional agricultural products and foods having a beneficial impact on health", Ministry of Agriculture, Forestry and Fisheries, Japan. 


\section{Conflicts of Interest}

The authors declare no conflicts of interest regarding the publication of this paper.

\section{References}

[1] Ashiuchi, M. (2010) Occurrence and Biosynthetic Mechanism of Poly-GammaGlutamic Acid. In: Hamano, Y., Ed., Amino-Acid Homopolymers Occurring in Nature, Springer Nature Switzerland AG, Basel, 77-93. https://doi.org/10.1007/978-3-642-12453-2_5

[2] Tanimoto, H. (2010) Food Applications of Poly-Gamma-Glutamic Acid. In: Hamano, Y., Ed., Amino-Acid Homopolymers Occurring in Nature, Springer Nature Switzerland AG, Basel, 155-168. https://doi.org/10.1007/978-3-642-12453-2_8

[3] Ashiuchi, M. (2011) Analytical Approaches to Poly- $\gamma$-Glutamate: Quantification, Molecular Size Determination, and Stereochemistry Investigation. Journal of Chromatography B Analytical Technologies in the Biomedical and Life Sciences, 879, 3096-3101. https://doi.org/10.1016/j.jchromb.2011.03.029

[4] Lee, E.H., Son, W.C., Lee, S.E. and Kim, B.H. (2013) Anti-Obesity Effects of Poly-Gamma-Glutamic Acid with or without Isoflavones on High-Fat Diet Induced Obese Mice. Bioscience, Biotechnology, and Biochemistry, 77, 1694-1702.

https://doi.org/10.1271/bbb.130253

[5] Park, J.H., Choi, J.C., Sung, M.H., Kang, J.H. and Chang, M.J. (2011) High Molecular Weight Poly-Gamma-Glutamic Acid Regulates Lipid Metabolism in Rats Fed a High-Fat Diet and Humans. Journal of Microbiology and Biotechnology, 21, 766-775. https://doi.org/10.4014/jmb.1104.04047

[6] Shin, E.J., Sung, M.J., Park, J.H., Yang, H.J., Kim, M.S., Hur, H.J. and Hwang, J.T. (2015) Poly-Gamma-Glutamic Acid Induces Apoptosis via Reduction of COX-2 Expression in TPA-Induced HT-29 Human Colorectal Cancer Cells. International Journal of Molecular Sciences, 16, 7577-7586. https://doi.org/10.3390/ijms16047577

[7] Choi, J.H., Pichiah, P.B., Kim, M.J. and Cha, Y.S. (2016) Cheonggukjang, a Soy-Bean Paste Fermented with B. licheniformis-67 Prevents Weight Gain and Improves Glycemic Control in High Fat Diet Induced Obese Mice. Journal of Clinical Biochemistry and Nutrition, 59, 31-38. https://doi.org/10.3164/jcbn.15-30

[8] Jeong, S.Y., Jeong, D.Y., Kim, D.S. and Park, S. (2018) Chungkookjang with High Contents of Poly-Gamma-Glutamic Acid Improves Insulin Sensitizing Activity in Adipocytes and Neuronal Cells. Nutrients, 10, 1588. https://doi.org/10.3390/nu10111588

[9] Ishikawa, A., Kishi, M. and Yamagami, K. (2009) Effect of Intake of Natto and Soybeans on Postprandial Blood Glucose Levels in Healthy Adults. Journal of Urban Living and Health Association, 53, 257-260.

[10] Miwa, H., Hiyama, C. and Yamamoto, M. (1985) High-Performance Liquid Chromatography of Short- and Long-Chain Fatty Acids as 2-Nitrophenylhydrazides. Journal of Chromatography $A, 321,165-175$. https://doi.org/10.1016/S0021-9673(01)90433-9

[11] Miwa, H., Yamamoto, M., Nishida, T., Nunoi, K. and Kikuchi, M. (1987) High-Performance Liquid Chromatographic Analysis of Serum Long-Chain Fatty Acids by Direct Derivatization Method. Journal of Chromatography B Biomedical Sciences and Applications, 416, 237-245. https://doi.org/10.1016/0378-4347(87)80507-8 
[12] Müller, M., Canfora, E.E. and Blaak, E.E. (2018) Gastrointestinal Transit Time, Glucose Homeostasis and Metabolic Health: Modulation by Dietary Fibers. $\mathrm{Nu}$ trients, 10, E275. https://doi.org/10.3390/nu10030275

[13] Thondre, P.S., Shafat, A. and Clegg, M.E. (2013) Molecular Weight of Barley Beta Glucan Influences Energy Expenditure, Gastric Emptying and Glycaemic Response in Human Subjects. British Journal of Nutrition, 110, 2173-2179. https://doi.org/10.1017/S0007114513001682

[14] Torsdottir, I., Alpsten, M., Holm, G., Sandberg, A.S. and Tölli, J. (1991) A Small Dose of Soluble Alginate-Fiber Affects Postprandial Glycemia and Gastric Emptying in Humans with Diabetes. Journal of Nutrition, 121, 795-799. https://doi.org/10.1093/jn/121.6.795

[15] Holst, J.J., Gribble, F., Horowitz, M. and Rayner, C.K. (2016) Roles of the Gut in Glucose Homeostasis. Diabetes Care, 39, 884-892.

https://doi.org/10.2337/dc16-0351

[16] Blaak, E.E., Antoine, J.M., Benton, D., Björck, I., Bozzetto, L., Brouns, F., Diamant, M., Dye, L., Hulshof, T., Holst, J.J., Lamport, D.J., Laville, M., Lawton, C.L., Meheust, A., Nilson, A., Normand, S., Rivellese, A.A., Theis, S., Torekov, S.S. and Vinoy, S. (2012) Impact of Postprandial Glycaemia on Health and Prevention of Disease. Obesity Reviews, 13, 923-984.

https://doi.org/10.1111/j.1467-789X.2012.01011.x

[17] Bergman, R.N. and Ader, M. (2000) Free Fatty Acids and Pathogenesis of Type 2 Diabetes Mellitus. Trends in Endocrinology \& Metabolism, 11, 351-356. https://doi.org/10.1016/S1043-2760(00)00323-4

[18] Stich, V. and Berlan, M. (2004) Physiological Regulation of NEFA Availability: Lipolysis Pathway. Proceedings of the Nutrition Society, 63, 369-374. https://doi.org/10.1079/PNS2004350

[19] Boden, G. (1997) Role of Fatty Acids in the Pathogenesis of Insulin Resistance and NIDDM. Diabetes, 46, 3-10. https://doi.org/10.2337/diab.46.1.3

[20] Paolisso, G., Tataranni, P.A., Foley, J.E., Bogardus, C., Howard, B.V. and Ravussin, E. (1995) A High Concentration of Fasting Plasma Non-Esterified Fatty Acids Is a Risk Factor for the Development of NIDDM. Diabetologia, 38, 1213-1217. https://doi.org/10.1007/BF00422371

[21] Pankow, J.S., Duncan, B.B., Schmidt, M.I., Ballantyne, C.M., Couper, D.J., Hoogeveen, R.C. and Golden, S.H. (2004) Fasting Plasma Free Fatty Acids and Risk of Type 2 Diabetes: The Atherosclerosis Risk in Communities Study. Diabetes Care, 27, 77-82. https://doi.org/10.2337/diacare.27.1.77

[22] Kuwahara, K., Honda, T., Nakagawa, T., Yamamoto, S., Hayashi, T. and Mizoue, T. (2017) Body Mass Index Trajectory Patterns and Changes in Visceral Fat and Glucose Metabolism Before the Onset of Type 2 Diabetes. Scientific Reports, 7, Article No. 43521. https://doi.org/10.1038/srep43521

[23] Reaven, G.M. (2011) The Metabolic Syndrome: Time to Get Off the Merry-Go-Round? Journal of Internal Medicine, 269, 127-136. https://doi.org/10.1111/j.1365-2796.2010.02325.x

[24] Sugawara, A. and Sone, H. (2011) Evidence for the Diagnosis of Metabolic Syndrome in Japan. The Japanese Journal of Nutrition and Dietetics, 69, 205-213. https://doi.org/10.5264/eiyogakuzashi.69.205

[25] Hara, T., Kimura, I., Inoue, D., Ichimura, A. and Hirasawa, A. (2013) Free Fatty Acid Receptors and Their Role in Regulation of Energy Metabolism. Reviews of Physiology, Biochemistry and Pharmacology, 164, 77-116. 
https://doi.org/10.1007/112_2013_13

[26] Kimura, I., Ozawa, K., Inoue, D., Imamura, T., Kimura, K., Maeda, T., Terasawa, K., Kashihara, D., Hirano, K., Tani, T., Takahashi, T., Miyauchi, S., Shioi, G., Inoue, H. and Tsujimoto, G. (2013) The Gut Microbiota Suppresses Insulin-Mediated Fat Accumulation via the Short-Chain Fatty Acid Receptor GPR43. Nature Communications, 4, Article No. 1829. https://doi.org/10.1038/ncomms2852 